\title{
Optimal Direct Load Control of Renewable Powered Small Cells: Performance Evaluation and Bounds
}

\author{
Nicola Piovesan, Marco Miozzo, Paolo Dini \\ CTTC/CERCA, Av. Carl Friedrich Gauss, 7, 08860, Castelldefels, Barcelona, Spain \\ \{npiovesan, mmiozzo, pdini\}@cttc.es
}

\begin{abstract}
In this paper, we propose an optimal direct load control of renewable powered small base stations based on Dynamic Programming. The optimization is represented using Graph Theory and the problem is stated as a Shortest Path problem. The proposed optimal algorithm is able to adapt to the varying conditions of renewable energy sources and traffic demands. We analyze the optimal ON/OFF policies considering different energy and traffic scenarios. Then, we evaluate network performance in terms of system drop rate and grid energy consumption. The obtained results are compared with a greedy approach. This study allows to elaborate on the behavior and performance bounds of the system and gives a guidance for approximated policy search methods.
\end{abstract}

Index Terms-Mobile Networks, Energy Sustainability, Optimal Control, Demand Response, Dynamic Programming, Direct Load Control

\section{INTRODUCTION}

Recently, cellular networks have witnessed an impressive increase in mobile user demand. This is due to the introduction of new services that require reliable and fast connectivity, as well as the increase in the number of connected devices, including machines. In fact, the data traffic is augmenting at a rate of approximately 1.5 to 2 times per year, while the emergence of machine-type communication and IoT will lead to 29 billion connected devices in 2022 [1]. Therefore, the fifth generation mobile network $(5 \mathrm{G})$ is expected to support 1000 times more data volume per unit area, 100 more user data rate, 1000 more connected devices, 10 times longer battery life and 5 times reduced end-to-end latency than 4G [2].

A new architecture and new network deployments are, thus, necessary to meet those requirements. Massive deployment of small base stations (SBSs) represents the most promising architecture to meet the high capacity demand of mobile networks. However, the deployment of such network elements will lead to an increase in the energy consumption of the network that will cause negative economic and environmental impacts. Predictions confirm that, if no actions are taken, the greenhouse gas (GHG) emissions per capita for Information and Communication Technology (ICT) will increase from 100 $\mathrm{kg}$ in 2007 to $130 \mathrm{~kg}$ in 2020, globally [3]. Moreover, the high energy consumption of the cellular network forces the operators to pay high bills, which constitute half of their operating expenditures (OPEX) [4]. Total global energy consumption by all mobile networks was approximately $120 \mathrm{TWh}$ in 2010, resulting in energy costs of $\$ 13$ billion [5]. Although

(C) 2018 IEEE this is not a recent estimate, it represents a lower bound for the actual values due to the increasing price of energy. Therefore, there is a need for controlling and mitigating the energy consumption in the next generation cellular networks.

The reduced energy requirements of small base stations encourage the use of renewable energy sources (RES) as distributed power suppliers. The adoption of renewable energy in modern mobile networks will lead to (i) a reduction of the grid energy consumption, (ii) a reduction of the carbon footprint of ICT and (iii) savings on the energy bills from the network operators [6].

The introduction of RES entails an intermittent and erratic energy budget for the communication operations of the SBSs. Therefore, Demand Response (DR) is fundamental to properly manage energy inflow and spending, based on the traffic demand. Considering the high number of SBSs in the system, distributed self-organizing techniques represents a viable solution that can enable intelligent energy management policies, such as Direct Load Control [7]. An optimal switch ON-OFF problem has been solved in [8] by using a two-stage DP algorithm. In particular, the BSs' on-off states are optimized in the first stage, and the active BSs' resource blocks are allocated iteratively in the second stage. However, the problem has been stated for a single-tier architecture.

In our previous work [9], a two-tier architecture with hybrid power suppliers is introduced: macro base stations (MBSs) reside in the first tier to provide baseline coverage and capacity and are powered by the electrical grid, whereas SBSs operate in the second tier to provide capacity enhancement and are supplied by solar panels plus batteries. The data traffic offloaded by the SBSs has higher spectral efficiency and allows a reduction of the energy drained from the grid. An optimal direct load control of renewable powered SBSs based on dynamic programming, is proposed. The DP optimization is represented using Graph Theory and the problem is stated as a shortest-path search. The Label Correcting Method is used to explore the graph and find the optimal ON/OFF policy for the SBSs. DP has the key property of applying optimal control as a trade-off between the present and the future costs. This is a fundamental feature in our scenario, to prevent SBSs blackout during periods with low renewable energy arrivals and high traffic demands. In this paper, we extend our previous work by analyzing the optimal policies considering different energy harvesting and traffic conditions. The obtained policies are also compared with a greedy approach. Finally, we evaluate 
the network performance in terms of system drop rate and grid energy consumption.

The remainder of the paper is organized as follows. In Section II we present the system model, whereas the optimization problem is formulated in Section III. In Section IV we analyze the optimal policies for different energy harvesting and traffic scenarios and we discuss some performance results. Finally, in Section $\mathrm{V}$ we draw our conclusions.

\section{SySTEM MODEL}

The Radio Access Network (RAN) is represented as a set of clusters. Each of them is composed of 1 macro base station (MBS) and $C$ small base stations (SBSs). Each SBS is powered by a solar panel and it can store energy into a battery. On the contrary, the MBS is connected to the electrical grid. The SBSs implement an intelligent energy management system that decides their operative state. The two feasible operative states are: (i) ON, where the SBS serves the users in its coverage area, and (ii) OFF, where the SBS is in an energy saving mode and its users are handed over to the MBS. The state of all the $C$ SBSs at time $t$ is described by the vector $\boldsymbol{S}_{t}=\left[S_{t}^{(1)}, S_{t}^{(2)}, \ldots, S_{t}^{(C)}\right]$. Each element $S_{t}^{(i)}$, with $i=1, \ldots, C$, is defined as follows:

$$
S_{t}^{(i)}= \begin{cases}0, & \text { if } i \text {-th SBS is OFF } \\ 1, & \text { if } i \text {-th SBS is ON }\end{cases}
$$

The energy harvested by the SBSs at time $t$ is indicated by the vector $\boldsymbol{E}_{t}=\left[E_{t}^{(1)}, E_{t}^{(2)}, \ldots, E_{t}^{(C)}\right]$, while the amount of energy stored in the SBSs batteries at time $t$ is indicated by the vector $\boldsymbol{B}_{t}=\left[B_{t}^{(1)}, B_{t}^{(2)}, \ldots, B_{t}^{(C)}\right]$.

The energy consumption of the BS is approximated by the linear function $P=P_{0}+\beta \rho$, where $P_{0}$ is the baseline power consumption and $\rho \in[0,1]$ is the normalized traffic load. The typical values of these parameters are $P_{0}^{\mathrm{MBS}}=750 \mathrm{~W}$, $\beta^{\mathrm{MBS}}=600$ for MBSs and $P_{0}^{\mathrm{SBS}}=105.6 \mathrm{~W}, \beta^{\mathrm{SBS}}=39$ for SBSs. This model is supported by real measurements and closely matches the real power profile of BSs [10].

We consider a LTE RAN with a transmission bandwidth $B W$ divided into $R$ resource blocks (RBs) of 1 msec per $180 \mathrm{kHz}$ each [11]. The traffic level of the SBSs at time $t$ is indicated by the traffic load vector $\rho_{t}=\left[\rho_{t}^{(1)}, \rho_{t}^{(2)}, \ldots, \rho_{t}^{(C)}\right]$. If the SBS $i$ is OFF at time $t$, its users are managed by the MBS and we assume that the SBS can be entirely switched OFF (e.g., $P=0$ ). However, the MBS may have reached its capacity limit at that time instant (i.e. cannot allocate any RB to users) and may drop part of the handed over users. This situation is defined as system outage.

\section{Optimization PROBLEM}

The periodicity of the traffic demand and the energy arrivals leads to a cyclic evolution of the system. At every cycle $t$, a centralized controller computes the optimal state configuration of the SBSs in the cluster.

This sequential decision making process is modeled as a DP optimization problem. The objective is to minimize the grid energy consumed by the MBS and the traffic drop rate of the system. Since there is a linear relation between the energy consumption and the BS load, the objective is converted into the minimization of the MBS load over a given time horizon, by offloading the traffic to the renewable powered SBSs. Furthermore, a threshold $B_{\text {th }}$ on the battery level is introduced to prevent damages to the storage devices [12].

The optimization problem is formulated as follows:

$$
\begin{gathered}
\min _{\left\{\boldsymbol{S}_{t}\right\}_{t=1, \ldots, K}} \sum_{t=1}^{K} f\left(\boldsymbol{S}_{t}, t\right) \\
B_{t}^{(i)}>B_{\mathrm{th}} \forall i .
\end{gathered}
$$

where $K$ is defined as time horizon of the optimization and corresponds to the number of times the control is applied. Furthermore, the cost function $f\left(\boldsymbol{S}_{t}, t\right)$ is defined as follows:

$$
f\left(\boldsymbol{S}_{t}, t\right)=\frac{1}{2}\left[w_{1} \cdot \mathrm{L}\left(\boldsymbol{S}_{t}, t\right)+w_{2} \cdot \mathrm{D}\left(\boldsymbol{S}_{t}, t\right)\right]
$$

where

- $\mathrm{L}\left(\boldsymbol{S}_{t}, t\right)$, is the normalized load of the MBS given the SBSs states and the time instant $t$.

- $\mathrm{D}\left(\boldsymbol{S}_{t}, t\right)$ is the traffic drop rate of the system, given the state of the SBSs and the time instant $t$. Its value ranges from 0 (when all the traffic is served by the system) to 1 (when all the traffic is dropped by the system).

The two weights must always sum to one, i.e., $w_{1}+w_{2}=1$.

The battery levels of the SBSs are updated at each decision instant $t$, according to the following formula:

$$
\boldsymbol{B}_{t+1}=\min \left(\boldsymbol{B}_{t}+\boldsymbol{E}_{t}-\left(\left(P_{0}^{\mathrm{SBS}}+\beta^{\mathrm{SBS}} \boldsymbol{\rho}_{t}\right) \Delta_{t}, B_{\text {cap }}\right)\right)
$$

where $B_{\text {cap }}$ is the maximum battery capacity. The amount of energy exceeding the battery capacity cannot be stored and it is wasted.

The complexity of this optimization problem increases quasi-exponentially with the number of SBSs in the cluster and with the time horizon. In order to solve it in an efficient way, this problem has been graphically represented and subsequently transposed to a graph theory shortest-path problem in [9]. The proposed algorithm based on the Label Correcting Method has been used to obtain the results shown in the following section.

\section{RESUlTS ANALYSIS}

\section{A. Simulation Scenario}

We consider a square area with a side of $1 \mathrm{~km}$. The MBS is located at the center of the area and 3 SBSs are randomly positioned. The SBSs have a transmission power of $38 \mathrm{dBm}$, which corresponds to a coverage radius of $50 \mathrm{~m}$. The coverage areas of the SBSs do not overlap. The base stations have a transmission bandwidth of $5 \mathrm{MHz}$. Aggregated downlink traffic has been generated based on the traffic profiles defined in [13]. In particular, we consider three different weekly traffic profiles: Resident, Transport and Office. User traffic is based 


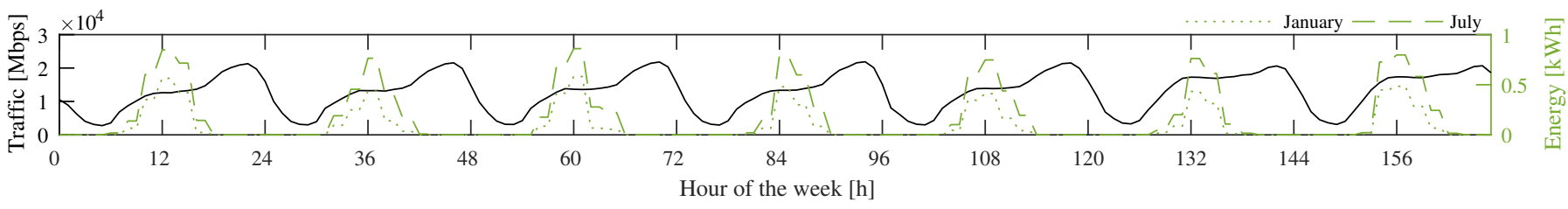

(a) Resident traffic profile

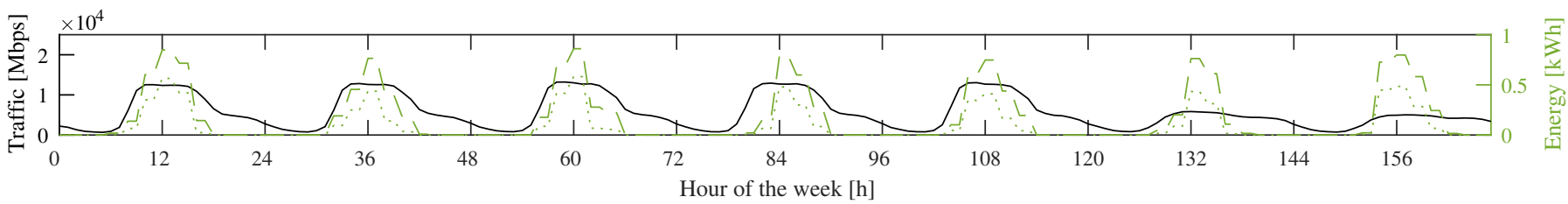

(b) Office traffic profile

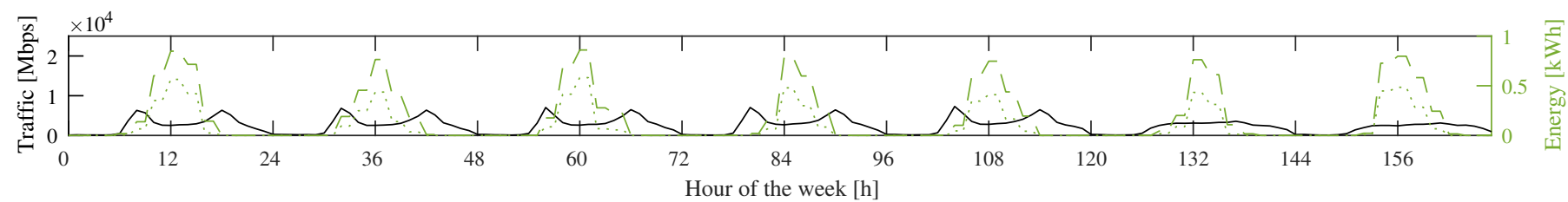

(c) Transport traffic profile

Fig. 1. Example of temporal variation of the traffic and energy harvesting process in a week of January and July. Scenario with 90 UEs per SBS with $50 \%$ of heavy users.

on the classification proposed in [10]. Heavy users have a data volume of $900 \mathrm{MB} / \mathrm{h}$, whereas ordinary users have a data volume of $112.5 \mathrm{MB} / \mathrm{h}$. We underline that, with the considered approach, the traffic is described both in time (temporal variation during the week) and in space (spacial distribution in the area).

As for the RES system, we consider the Panasonic N235B solar modules, which have single cell efficiencies of about $21 \%$ delivering about $186 \mathrm{~W} / \mathrm{m}^{2}$. Each SBS is equipped with an array of $16 \times 16$ solar cells (i.e. $4.48 \mathrm{~m}^{2}$ ). The battery size is $2 \mathrm{kWh}$ (panel and battery sizes have been chosen so that SBS batteries can be replenished in a full winter day). Realistic energy harvesting traces are obtained using the SolarStat tool [14], considering the city of Los Angeles. All the simulations have been performed considering a generic week of January and July, in order to highlight the differences between a generic winter (low energy arrivals) and a summer month (high energy arrivals). Moreover, the coordinator takes actions with a time step of 1 hour, considering a time horizon of 21 hours, as described in [9]. In the analysis here presented, we have assigned the same importance to the energy consumption and the system drop rate, when computing the optimal policy. Therefore, the weights in equation (3) have been set to $w_{1}=w_{2}=0.5$.

The optimal approach is compared with a greedy algorithm that operates by turning OFF the SBSs when their battery levels go below the threshold and turning them ON when the level is above it.

\section{B. Optimal policies}

An example of the temporal variation of the traffic and the harvested energy arrival processes during a week is shown in
Fig. 1. The traffic requests in considered areas differ both in terms of temporal distribution and magnitude. In particular, the Resident area is the most demanding, with a weekly aggregated traffic of $2.17 \mathrm{~TB}$. The lowest traffic demand is experienced in the Transport area, with a weekly aggregated traffic of $0.41 \mathrm{~TB}$. The three traffic profiles and the two energy arrival processes are not always time correlated. In fact, in the case of Resident traffic, the peak of the demand is at $10 \mathrm{pm}$. In the case of Office traffic, it is at 11 am on the weekdays and at midday on the weekends. Finally, in the Transport case we have two peaks during the weekdays, at 8 am and $6 \mathrm{pm}$, while on the weekends there is a single peak at $5 \mathrm{pm}$. From the energy side, the peak of energy arrivals is always between midday and $1 \mathrm{pm}$ for the two considered months. This confirms the necessity of taking into account present and future costs when the optimal control is applied.

The daily average switch OFF rate of the SBSs for the optimal and the greedy policy is reported in Fig. 2 and Fig. 3 , respectively. The three traffic profiles and the months of January and July are depicted. We consider a high-traffic intensity involving 90 UEs (50\% heavy) represented by solid lines, and a low-traffic intensity with 10 UEs (20\% heavy) indicated with dashed lines.

In Fig. 2 we observe that the number of SBSs in OFF is generally higher during night hours. This fact is due to the scarce availability of the energy and the low traffic demand during the night. More in detail, we can notice that high-traffic intensity and low energy arrivals (January) result in longer and more intensive switch OFF periods during the night. For the Resident profile (Fig. 2a) the switch OFF rate in a typical week of July is intensive between 3 am and 6 am (low-traffic 


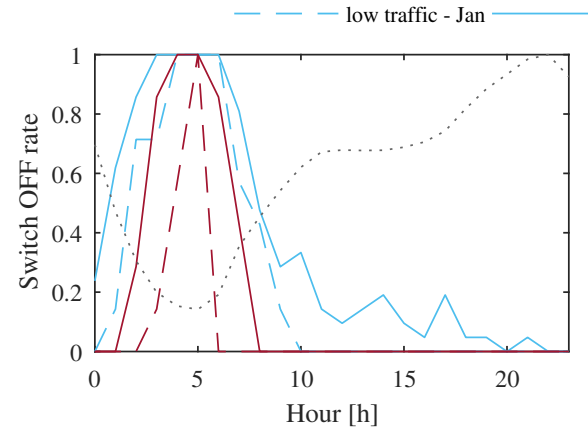

(a) Resident traffic

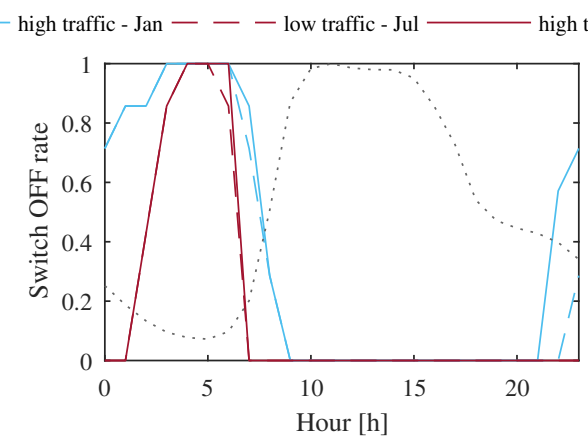

(b) Office traffic

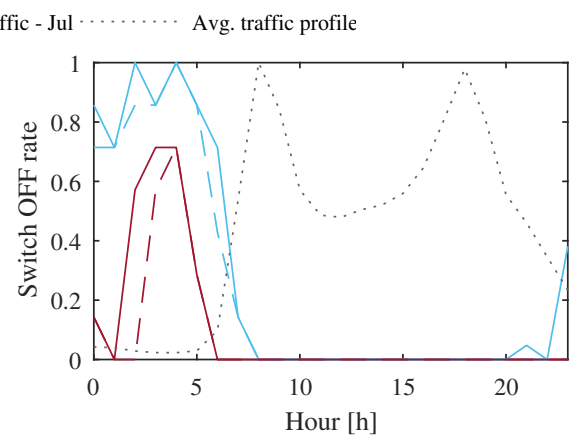

(c) Transport traffic

Fig. 2. Daily average switch OFF rate for the optimal algorithm. Simulations on the Resident, Office and Transport traffic profile for a week of January and July. The scenario with 10 UEs ( $20 \%$ heavy) is indicated as low traffic, whereas the scenario with 90 UEs (50\% heavy) is indicated as high traffic.

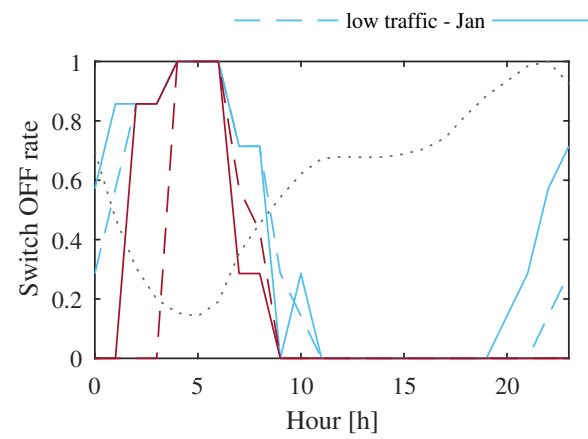

(a) Resident traffic

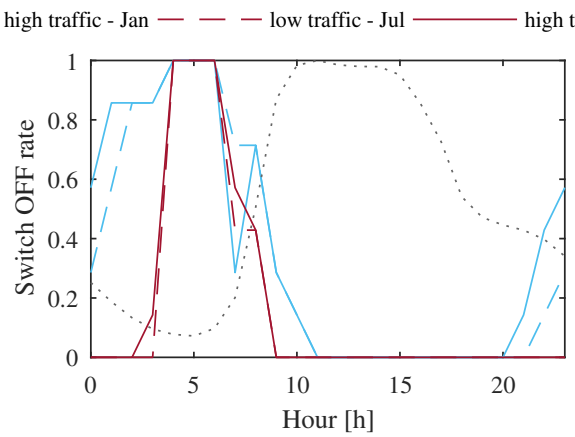

(b) Office traffic

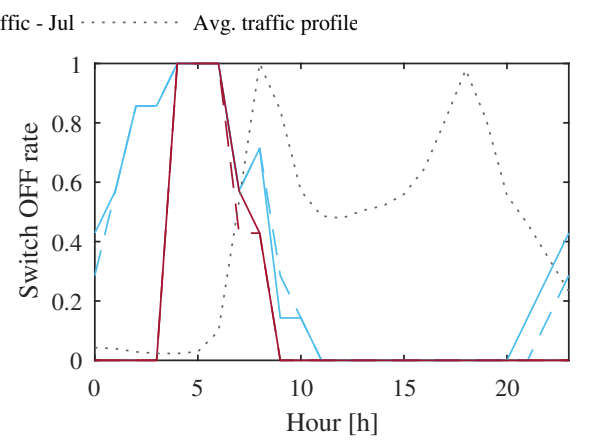

(c) Transport traffic

Fig. 3. Daily average switch OFF rate for the greedy algorithm. Simulations on the Resident, Office and Transport traffic profile for a week of January and July. The scenario with 10 UEs (20\% heavy) is indicated as low traffic, whereas the scenario with 90 UEs (50\% heavy) is indicated as high traffic.

case) and from 2 am to 8 am (high-traffic case). In a week of January, the switch OFF rate is intensive from 1 am to 10 am (low-traffic case). In the case of Office (Fig. 2b) and Transport (Fig. 2c) profiles, these intensive night OFF periods are less influenced by the total number of UEs served by the SBSs. This is due to the low magnitude of the total traffic demand experienced in these areas.

In Fig. 3 we observe that the switch OFF rate is intensive also in periods of high traffic demand. The greedy algorithm takes immediate decisions without considering any future evolution of the traffic and energy arrival processes. In this way, a SBS always consumes the available energy and then it remains in an OFF state until the harvested energy is sufficient to return operative. On the contrary, the optimal policy turns OFF a SBS in an intelligent way, by considering future evolutions of the traffic and energy arrivals. Therefore, it saves energy during low traffic periods (e.g. night hours) to maintain ON the SBS during high traffic peaks, which may correspond to scarce energy arrivals. For instance, let's consider the Resident profile with high-traffic intensity in January, where the peak of the traffic demand is at $10 \mathrm{pm}$. The greedy algorithm switch OFF rate is 0.5 at $10 \mathrm{pm}$ (Fig. 3a), whereas it is zero during the daytime (i.e., from 11 am to $7 \mathrm{pm}$ ). In fact, the SBSs are immediately using the available energy during the day, and then switching OFF in the evening due to scarce energy availability. On the contrary, the optimal switch OFF rate (Fig. 2a) is almost zero during the traffic peak hours and it is not null during the daytime. This behavior indicates that some SBSs are switched OFF during the day to save the necessary energy to satisfy the traffic peak in the evening.

\section{System outage}

In this section, we analyze the system outage measured as the percentage of the traffic dropped in the system. We present the case of Resident profile only, since the others have similar performance. The percentage of the traffic dropped in a week is reported in Fig. 4 for a number of UEs ranging from 10 to $90(50 \%$ of them are heavy users).

The optimal policy succeeds in delivering all the traffic requested and the system does not experience any outage in almost every studied situation. In January, however, some traffic is dropped starting from 60 UEs per SBS, reaching the maximum of $0.9 \%$ for 90 UEs. The greedy approach, on the other hand, always performs worse than the optimal policy. In particular, in January the traffic dropped is reaching $10 \%$ in the case of 90 UEs per SBS.

This phenomenon is confirmed in Fig. 5, where the average hourly traffic dropped is shown for a scenario with 90 UEs per SBS with $50 \%$ of heavy users. As for the optimal policy, the outage is concentrated in the morning (from 7 am to 10 am), afternoon $(5 \mathrm{pm})$ and night, with values that reach the 


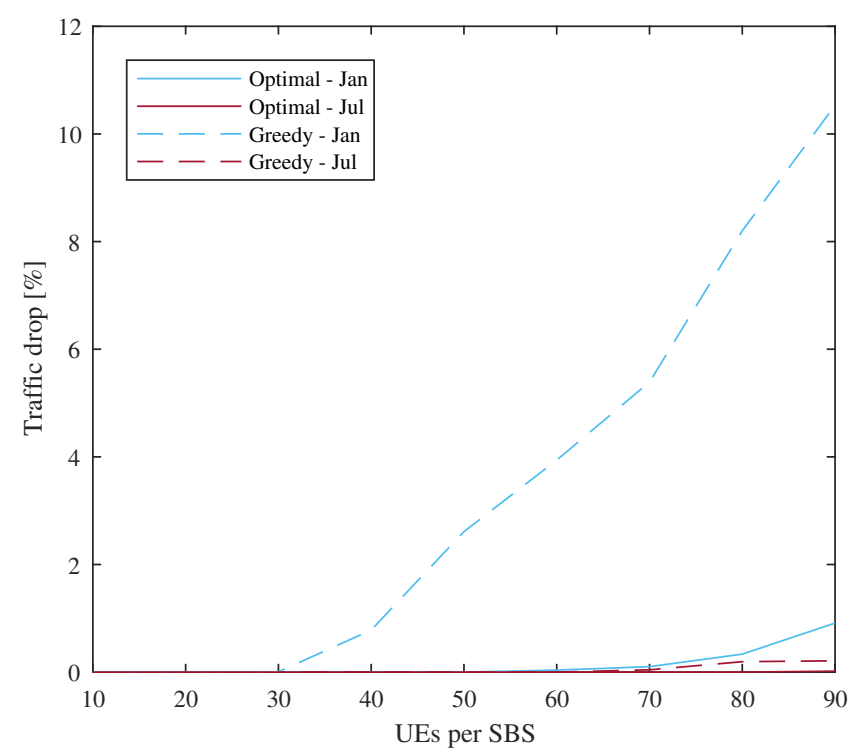

Fig. 4. Percentage of weekly traffic request not serviced for both the greedy and optimal algorithm in January and July. The $50 \%$ of the UEs are heavy users.

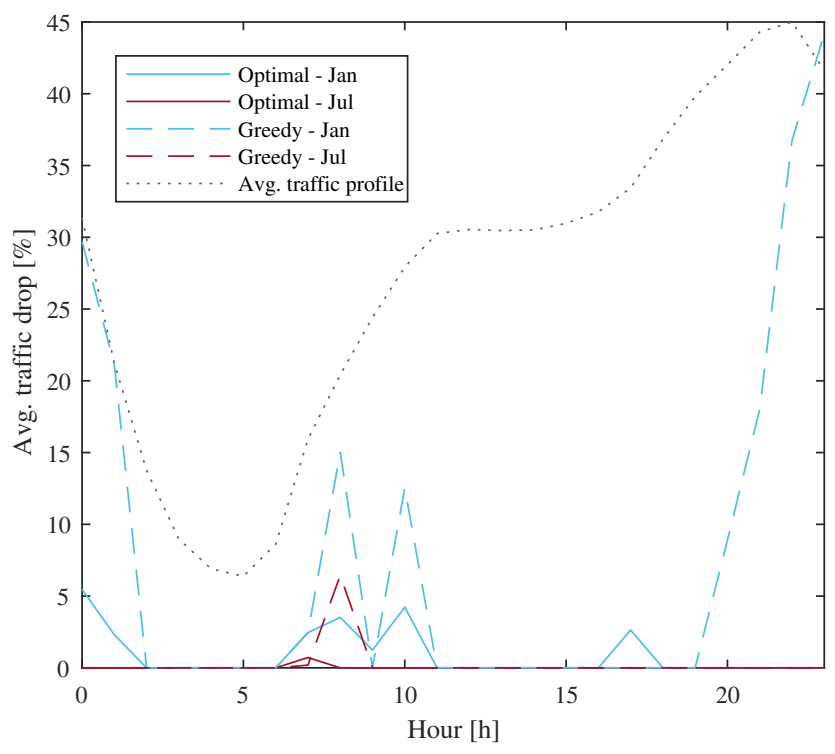

Fig. 5. Average hourly traffic drop for the optimal and the greedy algorithms in January and July. The traffic profile is Resident and every SBS has 90 UEs in its coverage area; $50 \%$ of them are heavy users.

maximum value of $5 \%$ at midnight. The greedy approach, instead, causes system outage for longer periods and with higher values of the dropped traffic, which is reaching a maximum of $44 \%$ at $11 \mathrm{pm}$.

\section{Energy consumption}

The amount of the grid energy consumed by the MBS is shown is Fig. 6, varying the number of UEs in the coverage area of the SBSs. We consider two cases: a scenario with $20 \%$ and $50 \%$ of heavy users, respectively. In both scenarios, the grid energy consumption increases linearly with the number of UEs. The slope of the curves is higher for the scenario

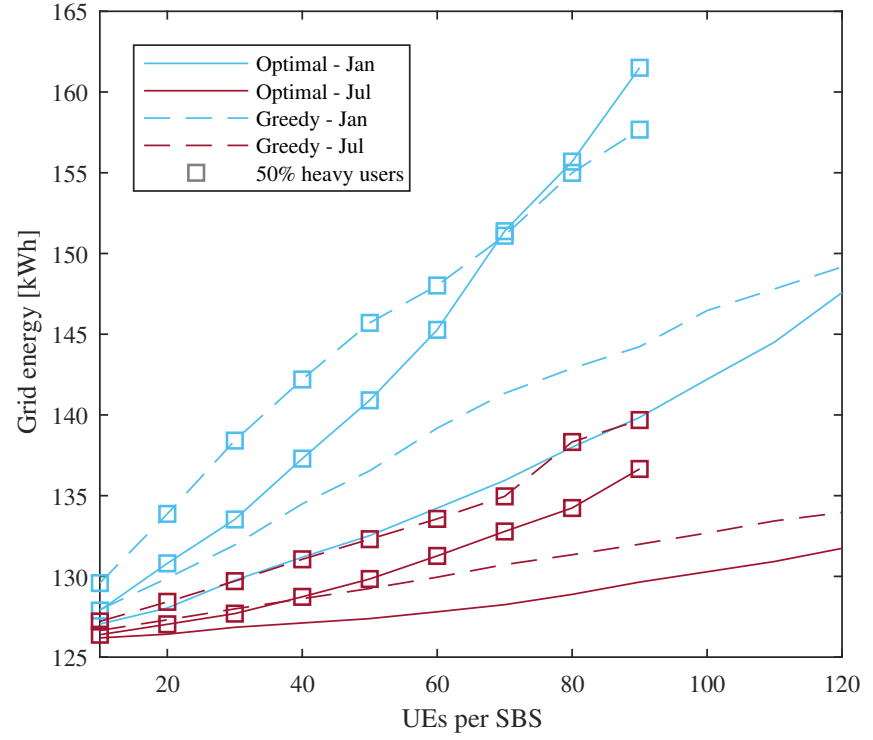

Fig. 6. Grid energy consumption for the optimal and the greedy algorithms in January and July, while increasing the number of UEs in the SBS area.

with $50 \%$ of heavy users since the traffic is increasing faster with the number of UEs. Grid energy consumption is higher during the winter months since the scarce availability of the renewable energy turns out into longer SBS sleeping periods and higher MBS operation.

The greedy approach presents higher values of the grid energy consumption than the optimal policy. However, in the case of January and with $50 \%$ of heavy users, we observe that the greedy approach has lower energy consumption for more than 70 UEs per SBS. This behavior is due to the fact that the system is heavily in outage and loses a considerable amount of traffic, as described in the previous subsection.

Finally, Fig. 7 reports the grid energy consumption for a week of July of different architecture scenarios. We compare a solution where MBS and SBSs are connected to the grid (also referred to as grid-only) with our scenario where SBSs are solely powered by solar panel plus battery (also referred to as EH SBS). The grid-only scenario consumes $190.3 \mathrm{kWh}$ in a week; deploying renewable powered SBSs saves $28 \%$ of the grid energy. Moreover, since the RES systems have been dimensioned for winter, the harvested energy may be abundant during summer and be discarded by the SBSs, i.e., it can neither be used for transmission nor stored in the battery. This redundant energy is concentrated during the peak hours of the energy arrival process (i.e., between midday and 2 pm). Considering that the SBSs may be connected through a power micro-grid, the excess energy can be used for ancillary services (e.g., light system) or shared to support the MBS operation, thus reducing its grid energy consumption. In fact, a grid energy saving of $38 \%$ is achieved, in case the MBS instantly uses the energy shared by the SBSs (also referred to as EH SBSs + energy sharing). However, the MBS might not be able to instantly consume the whole amount of the received energy, which would create instability in the micro-grid. For 


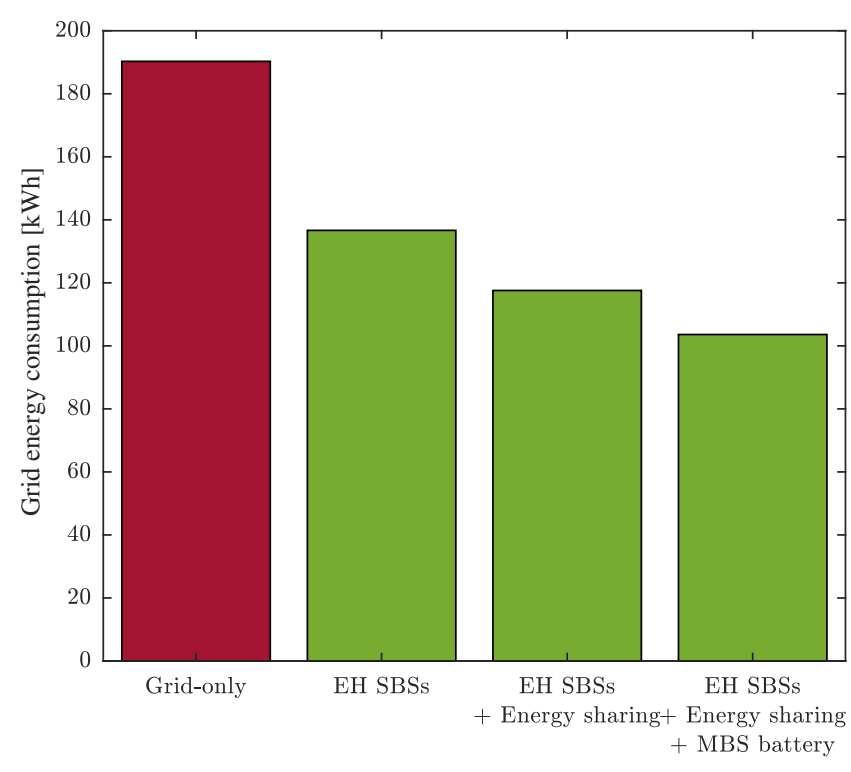

Fig. 7. Grid energy consumption for different deployment architectures during a week of July. The traffic profile is Resident and every SBS has 90 UEs in its coverage area; $50 \%$ of them are heavy users.

this reason, we consider the option of deploying a storage device at the MBS site (also referred to as EH SBSs + energy sharing + MBS battery). As a result, the MBS uses the shared energy when needed and saves $46 \%$ of the grid energy.

\section{CONCLUSIONS}

In this paper, we have introduced an optimal direct load control of renewable powered SBS in a two-tier mobile network. We have analyzed the optimal policies and their dependence on the traffic and the energy arrival process. We have compared the optimal approach with a greedy algorithm and analyzed the network performance in different scenarios. We have also introduced a new possibility of energy sharing among the network elements to reduce the dependence on the power grid and increase the energy savings.

From this analysis, we can draw the following conclusions. The different temporal behavior of the traffic and energy arrival processes highlights the necessity of deploying storage devices along with solar panels. Moreover, it is fundamental to properly manage the storage to maintain good network performance. The comparison between the optimal and the greedy approach shows that grid energy savings and traffic drop limitations are possible only if the control algorithm is able to forecast the evolution of the two processes. Finally, the analysis of the redundant energy shows that sharing energy among base stations may lead to considerable amount of grid energy savings.

\section{ACKNOWLEDGMENT}

This work has received funding from the European Union Horizon 2020 research and innovation programme under the Marie Sklodowska-Curie grant agreement No 675891 (SCAVENGE) and by the Spanish Government under project TEC2017-88373-R.

\section{REFERENCES}

[1] "Ericsson mobility report," Ericsson, Tech. Rep., June 2017. [Online]. Available: https://www.ericsson.com/assets/local/mobilityreport/documents/2017/ericsson-mobility-report-june-2017.pdf

[2] A. Osseiran, V. Braun, T. Hidekazu, P. Marsch, H. Schotten, H. Tullberg, M. A. Uusitalo, and M. Schellman, "The foundation of the mobile and wireless communications system for 2020 and beyond: Challenges, enablers and technology solutions," in IEEE Vehicular Technology Conference (VTC Spring), Dresden, Germany, June 2013, pp. 1-5.

[3] "Ericsson energy and carbon report," Ericsson, Tech. Rep., November 2014. [Online]. Available: https://www.ericsson.com/assets/local/aboutericsson/sustainability-and-corporate-responsibility/documents/ericssonenergy-and-carbon-report.pdf

[4] H. Ghazzai, E. Yaacoub, A. Kadri, H. Yanikomeroglu, and M. S. Alouini, "Next-generation environment-aware cellular networks: Modern green techniques and implementation challenges," IEEE Access, vol. 4, pp. 5010-5029, September 2016.

[5] "Mobiles green manifesto 2012," GSMA, Tech. Rep., June 2012. [Online]. Available: https://www.gsma.com/publicpolicy/mobilesgreenmanifesto

[6] G. Piro, M. Miozzo, G. Forte, N. Baldo, L. A. Grieco, G. Boggia, and P. Dini, "Hetnets powered by renewable energy sources: Sustainable next-generation cellular networks," IEEE Internet Computing, vol. 17, no. 1, pp. 32-39, Jan 2013.

[7] G. Koutitas and L. Tassiulas, "Smart grid technologies for future radio and data center networks," IEEE Communications Magazine, vol. 52, no. 4, pp. 120-128, April 2014.

[8] J. Gong, J. S. Thompson, S. Zhou, and Z. Niu, "Base station sleeping and resource allocation in renewable energy powered cellular networks," IEEE Transactions on Communications, vol. 62, no. 11, pp. 3801-3813, 2014.

[9] N. Piovesan and P. Dini, "Optimal direct load control of renewable powered small cells: A shortest path approach," Internet Technology Letters, September 2017.

[10] G. Auer, O. Blume, V. Giannini, I. Godor, M. Imran, Y. Jading, E. Katranaras, M. Olsson, D. Sabella, P. Skillermark et al., "D2. 3: Energy efficiency analysis of the reference systems, areas of improvements and target breakdown," EARTH, vol. 20, no. 10, 2010.

[11] S. Sesia, M. Baker, and I. Toufik, LTE-the UMTS long term evolution: from theory to practice. John Wiley \& Sons, 2011.

[12] M. Mendil, A. D. Domenico, V. Heiries, R. Caire, and N. Hadjsaid, "Fuzzy Q-Learning based Energy Management of Small Cells Powered by the Smart Grid," in IEEE PIMRC, Valencia, Spain, 2016.

[13] F. Xu, Y. Li, H. Wang, P. Zhang, and D. Jin, "Understanding mobile traffic patterns of large scale cellular towers in urban environment," IEEE/ACM Transactions on Networking (TON), vol. 25, no. 2, pp. 11471161, 2017.

[14] M. Miozzo, D. Zordan, P. Dini, and M. Rossi, "Solarstat: Modeling photovoltaic sources through stochastic markov processes," in Energy Conference (ENERGYCON), 2014 IEEE International. IEEE, 2014, pp. 688-695. 